\title{
UN BUS COME SPAZIO PUBBLICO. MULTICULTURALISMO ED ETNOGRAFIA URBANA A MILANO
}

\author{
A bus as a public space. \\ Multiculturalism and Urban Ethnography in Milan
}

\author{
Paola Briata* \\ Massimo Bricocoli* \\ Martina Bovo*
}

Riassunto. II contributo presenta gli esiti di un'attività didattica e di ricerca sul campo condotta presso il Politecnico di Milano a ridosso di un tema chiave: il ruolo degli spazi pubblici urbani come supporto per la negoziazione delle differenze e nell'ingaggiare diversi individui e popolazioni in situazioni di confronto e coesistenza. Un'esplorazione di tipo etnografico ha messo sotto osservazione un mezzo di trasporto pubblico quale spazio pubblico "compresso". Il filobus 90/91 a Milano è stato assunto quale terreno di osservazione privilegiato sui rapidi processi di cambiamento sociale e demografico che hanno investito Milano negli ultimi anni entro una prospettiva di ricerca sulla dimensione quotidiana del multiculturalismo.

Parole chiave: multiculturalismo; mobilità; trasporto pubblico; Italia.

\begin{abstract}
The paper draws on a research and teaching project carried out with an international class of students in Urban Ethnography in the MSC in Urban Planning and Policy at Politecnico di Milano (Italy). A particular focus of the project was on exploring the role played by public spaces in supporting the coexistence of a multitude of strangers in the city through the continuous negotiation of diversity and difference. In the field work, spatial and social dynamics occurring in a particular and 'compressed' public space - the 90-91 trolley-bus circle-line in Milan - are explored and discussed as a space of confrontation in a perspective of daily multiculturalism.
\end{abstract}

Keywords: multiculturalism; mobility; public transport; Italy.

\footnotetext{
* Dipartimento di Architettura e Studi Urbani, Politecnico di Milano. Milano, Italia.
} 


\section{Introduzione}

Questo contributo discute gli esiti di un'attività didattica e di ricerca sul campo condotta nell'ambito di un corso di Urban Ethnography nel Master of Science in Urban Planning and Policy Design e in Architecture presso il Politecnico di Milano. II corso è stato costruito a ridosso di un tema chiave: il ruolo degli spazi pubblici urbani come supporto per la negoziazione delle differenze. In particolare, abbiamo assunto l'ipotesi che nella dimensione quotidiana delle presenze e delle interazioni tra i corpi, gli spazi pubblici giochino un ruolo essenziale nell'ingaggiare diversi individui e popolazioni in situazioni di confronto e coesistenza.

Se in Italia la letteratura in campo urbano ha concentrato l'attenzione sugli impatti dell'immigrazione, soprattutto con riferimento ai quartieri nei quali si registra una concentrazione di residenti stranieri o di attività commerciali etnicamente connotate, il tema degli spazi di multiculturalismo quotidiano ${ }^{1}$ nei quali si dispiega la coesistenza di gruppi sociali, culturali ed economici è ancora poco esplorato. Nello sviluppare una riflessione in tal senso, il corso ha mobilitato due nozioni che ci paiono particolarmente interessanti e fertili: il concetto di convivenza tra estranei - strangers - sviluppato da $\mathrm{Amin}^{2}$, e quello di cosmopolitan canopy proposto da Anderson ${ }^{3}$.

Dopo una prima fase di introduzione teorica e di discussione più generale del tema e delle questioni, una parte consistente del corso è stata dedicata a un'esercitazione sul campo. Si è scelto di considerare quale spazio sul quale svolgere un'esplorazione di tipo etnografico un mezzo di trasporto pubblico urbano: il filobus 90/91 a Milano (Fig. 1). Si tratta dell'unico servizio di trasporto attivo 24 ore su 24 e il suo percorso, lungo un ampio anello circolare attorno alla porzione più centrale della città, delinea una sorta di confine simbolico e materiale tra centro e periferia. Si tratta di un mezzo utilizzato in modo intensivo da persone molto diverse in termini di background etnico, economico, sociale e culturale e del quale i media forniscono spesso rappresentazioni fortemente stigmatizzate. L'itinerario e la tabella oraria assai estesa ne fanno un punto di osservazione privilegiato sui rapidi processi di cambiamento sociale e demografico che hanno investito Milano negli ultimi anni.

La definizione di spazio pubblico urbano è stata in questo senso "ampia" e ha lasciato a ciascuno studente la scelta di perimetrare il proprio campo di osservazione concentrandosi sul veicolo e sulle pratiche che vi hanno luogo, oppure di estendere l'osservazione alle aree che il veicolo attraversa o in cui sosta.

\footnotetext{
WISE, Amanda, VELAYUTHAM, Selvaraj (eds.). Everyday multiculturalism.

AMIN, Ash. Land of Strangers.

3 ANDERSON, Elijah. The Cosmopolitan Canopy. Race and Civility in Everyday Life.
} 
Il contributo è organizzato come segue: il §1 si sofferma sugli aspetti metodologici della ricerca etnografica, sottolineando la rilevanza del coinvolgimento di studenti provenienti da tutto il mondo. Nel § 2 si propone una rassegna della letteratura che ha guidato la ricerca sul campo illustrata anche come primo spunto di "posizionamento" e approfondimento teorico agli studenti. Nel § 3 si inquadrano il contesto italiano e milanese in materia di immigrazione in termini di consistenza del fenomeno, discorso pubblico e politiche. II § 4 ricostruisce le narrazioni più comuni sulla 90/91, così come le reazioni dell'amministrazione ai processi di stigmatizzazione che caratterizzano questo filobus. I § 5 e 6 illustrano alcuni dei risultati più significativi della ricerca sul campo e vengono infine tratte delle conclusioni.

\section{Figura 1 - La linea 90/91 a Milano}

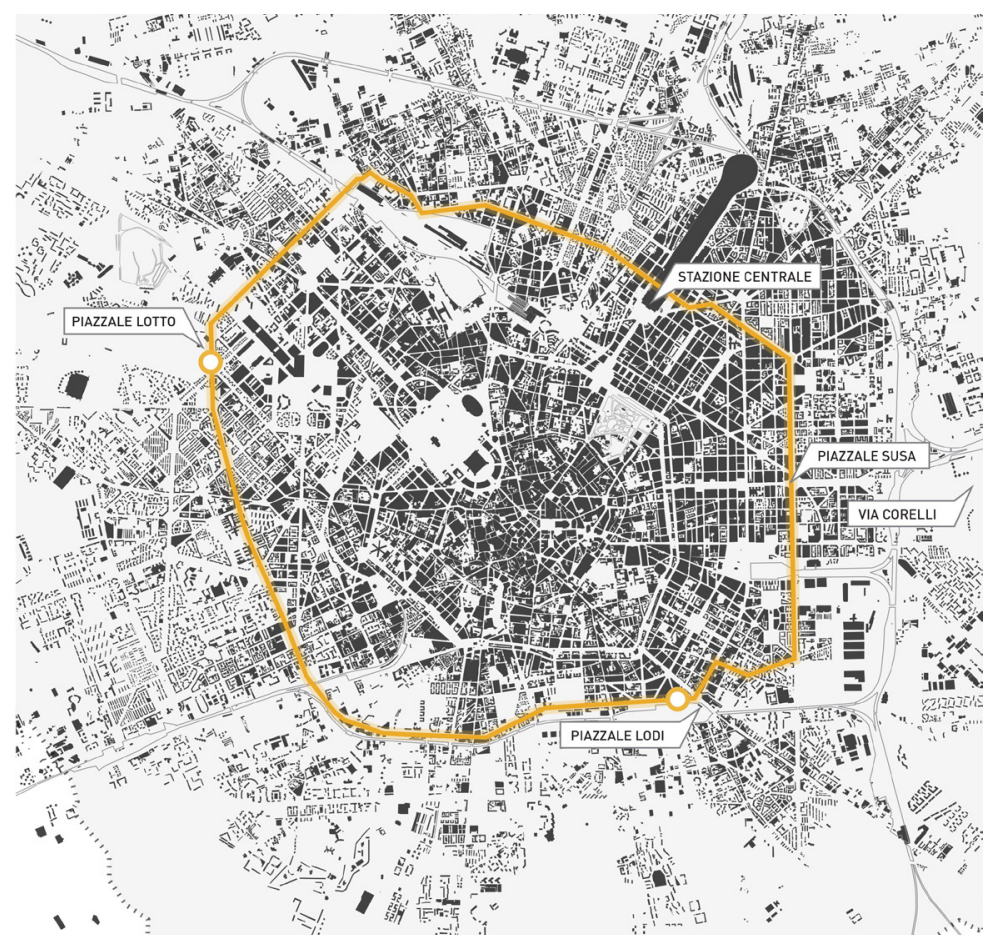

Fonte: elaborazione Martina Bovo.

\section{Camminare attraverso. Assumere la 90-91 quale punto di vista sulla città}

Il corso di Urban Ethnography giunge a valle di un corso di studi pluriennale e si rivolge a studenti che hanno una formazione già ricca in termini di strumenti di analisi e di ricerca urbana. I motivi di interesse e gli argomenti che hanno 
sostenuto negli anni questa proposta formativa sono da individuare proprio in una riflessione sugli strumenti di analisi dell'urbanistica e dell'architettura e che sollecita a "tornare" allo sguardo diretto, all'osservazione e all'esperienza degli spazi urbani quali fonti legittime e rilevanti di conoscenza. II richiamo è qui al contributo di Patrick Geddes ${ }^{4}$ e alle sue sollecitazioni circa la formazione di chi è impegnato nel progetto urbano. Geddes propone che lo sguardo del planner sulla città si articoli attraverso la composizione di uno sguardo "dall'alto" e uno sguardo "attraverso" ${ }^{5}$. Il riferimento alla Camera obscura e alla Outlook tower che Geddes ideò e realizzò a Edimburgo è una buona metafora rispetto alla frequenza con cui le questioni che attraversano lo spazio della città sono generalmente analizzate e rappresentate a partire da un punto di vista che sta in alto e che ci predispone a una vista zenitale sull'organizzazione di spazi e persone. Così è pure, entro questa metafora, per le analisi quantitative e le rappresentazioni cartografiche che riportano dati statistici su una mappa. "Il planner geddesiano cammina per osservare e per raccontare ciò che ha

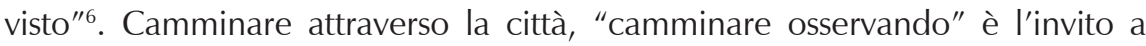
un'esplorazione talvolta tortuosa, ma mai casuale, un invito a un orientamento etnografico in cui l'osservazione diretta - se non anche partecipante - dei luoghi diventa strumento rilevante di conoscenza.

Rivolto a una classe internazionale composta da 25 studenti provenienti da paesi e continenti diversi, il corso si è connotato quale terreno incredibilmente ricco per un confronto tra background formativi e culturali diversificati. Per una scuola europea di architettura e urbanistica, la possibilità di un confronto diretto in aula tra formazioni e punti di vista ancorati a mondi culturali tanto differenti è un' occasione straordinaria per comprendere l'influenza delle diverse dimensioni - culturale, simbolica, storica e normativa - nella costruzione dei problemi che attingono all'organizzazione e al progetto dello spazio.

Negli ultimi due anni accademici (2016-2018), dopo una serie di lezioni introduttive circa la dimensione teorica e pratica che un orientamento etnografico può portare alla comprensione dei fenomeni urbani e dell'organizzazione dello spazio pubblico, è stato proposto di sviluppare la dimensione empirica del corso nella forma di un esercizio di osservazione diretta prolungata entro un campo che ogni partecipante ha potuto individuare, perimetrare e precisare a ridosso della 90/91. Una serie di seminari con interlocutori esterni ha consentito di articolare e mettere in evidenza le molteplici dimensioni che sono implicate attorno alla nozione di spazio pubblico e di coesistenza multiculturale con riferimento al trasporto pubblico. Federica Verona ha narrato la sua ricerca sulla linea del filobus 90/91 soffermandosi sulle narrazioni dei media; Martina Bovo

\footnotetext{
${ }^{4}$ GEDDES, Patrick. Cities in Evolution.

FERRARO, Giovanni. Rieducazione alla speranza. Patrick Geddes Planner in India 1914-1924.

6 Ibidem, p. 106.
} 
e Matilde Cassani hanno messo in evidenza la relazione tra flussi migratori e riconfigurazione dello spazio pubblico nella città italiana; Giovanni Vecchio ha raccontato il contributo che la mobilità urbana può dare allo sviluppo di risorse e capabilities individuali; Paola Piscitelli ha introdotto gli aspetti sociali e spaziali di guardare alle "persone come infrastruttura" sviluppato da AbduMaliq Simone”; un autista di lungo corso ha narrato la sua esperienza lavorativa e umana al volante di uno dei mezzi di trasporto più intensamente utilizzati in città ${ }^{\circ}$.

La restante parte del corso è stata dedicata a supportare un'attività individuale di osservazione diretta partecipante in corrispondenza di alcune domande di ricerca: com'è vissuta la 90/91 a ore diverse del giorno e della notte? Come avviene la negoziazione della differenza e come si riflette nell'uso di questo spazio "compresso"? È possibile considerare la 90-91 come un cosmopolitan canopy, un luogo dove "la manifestazione dell'accettazione pubblica di tutti da parte di tutti è particolarmente intensa, diventando una delle caratteristiche che definiscono un luogo"? ?9 Se sì, in che modo questa caratteristica può rappresentare una potenzialità per la città di Milano?

\section{La mobilità urbana in una prospettiva di multiculturalismo quotidiano}

Diversi filoni di letteratura nell'ambito delle scienze sociali sono stati utilizzati per costruire un background teorico di riferimento. Assunto quale oggetto di studio un mezzo di trasporto pubblico, gli studi qualitativi in materia di mobilità sono stati utili a definire lo stato dell'arte di questo campo d'indagine. D'altro lato, il taglio tematico sulla coesistenza di popolazioni molto diverse sulla 90/91 è stato supportato dagli studi sul multiculturalismo quotidiano e dal richiamo a quei tratti che Anderson segnala come rilevanti nella caratterizzazione di quei luoghi urbani che si configurano come cosmopolitan canopy, che garantiscono una dimensione di "lasco", di compresenza possibile e poco mediata tra individui sconosciuti e differenti.

Sul primo fronte, la letteratura in materia di pianificazione e trasporti vede il prevalere di indagini di matrice quantitativa che raccontano delle caratteristiche dei viaggiatori in termini di reddito, razza, etnia, genere o età. Negli ultimi anni la ricerca ha iniziato a spostarsi anche su questioni di natura qualitativa al fine di cogliere in modo più profondo i comportamenti dei viaggiatori e la dimensione sociale degli spostamenti. Un cosiddetto

\footnotetext{
SIMONE, AbdouMaliq. People as Infrastructure: Intersecting Fragments in Johannesburg.

8 Cfr. <https://federicaverona.com/tag/federica-verona/; <http://www.matildecassani.com/>; BOVO Martina, LIPPI, Chiara. The Receptive City; PISCITELLI, Paola. Mobile Urbanity in Southern Africa; VECCHIO, Giovanni. Democracy on the move? Bogotá's urban transport strategies and the access to the city.

9 ANDERSON, op. cit., p. 3.
} 
"mobility turn" caratterizza studi che hanno via via superato la dicotomia tra ricerca trasportistica e sociale, analizzando le relazioni sociali nel campo della mobilità e mettendo in relazione forme di trasporto e pattern di pratiche ed esperienze $^{10}$. Tuttavia, gli studi qualitativi in materia di mobilità e trasporti rimangono un campo d'indagine poco sviluppato ${ }^{11}$. Lo studio etnografico sugli autobus di lunga e breve percorrenza realizzato a Los Angeles da Fink ${ }^{12}$ è stato particolarmente utile per orientare il nostro lavoro. Si tratta di una ricerca che ha messo sotto osservazione gli spazi di transito come particolare tipo di spazio pubblico che espone i passeggeri al contatto con persone molto diverse. La proposta che abbiamo accolto è di guardare alla vita sugli autobus secondo un orientamento che considera la mobilità nelle sue diverse forme quale fattore fondamentale di trasformazione del nesso spazio-società ${ }^{13}$.

Un aspetto importante da sottolineare in una prospettiva internazionale è che in Italia i mezzi di trasporto pubblico urbano sono utilizzati in modo estensivo da un ampio range di popolazioni. Nella discussione in aula, i racconti di Clara Ebert su Rio de Janeiro, di Bilal El Ghoul su Beirut, di Vinita Wagh su Pune in India tra gli altri, hanno messo in evidenza come in alcuni paesi le condizioni di sovraffollamento, la frequenza di furti e aggressioni, la mancanza di comfort in condizioni climatiche avverse, la scarsa efficienza e affidabilità sono fattori che variamente condizionano l'utilizzo dei mezzi di trasporto pubblico; questi tratti di criticità tendono ad alimentare un'immagine negativa - quando non una vera e propria stigmatizzazione - ed il loro utilizzo è prevalente da parte dei ceti più poveri. A Milano, il sistema di trasporto pubblico è capillare e intensamente utilizzato. Se mai si dà un fenomeno di stigmatizzazione, questo è riferito solamente ad alcune linee e in particolare proprio alla 90/91. Si tratta dunque di un'eccezionalità e non della regola.

Sempre il lavoro di Fink ha fornito alcuni riferimenti che hanno restituito immagini suggestive. In particolare, il lavoro sulle "comunità sulle ruote" studiate da Nash ${ }^{14}$ che ha descritto la "normalità" e le sovversioni delle routine quotidiane di un mezzo di trasporto; gli studi sui voli aerei condotti da Zurcher" ${ }^{15}$ hanno introdotto l'immagine evocativa delle "condutture di persone", puntando l'attenzione sulle relazioni sociali in uno spazio compresso e fortemente regolato; il lavoro di Vannini ${ }^{16}$ propone di guardare al tempo di viaggio non come a uno "spazio morto" o a un'esperienza culturale neutra, perché i viaggiatori creano e trasformano lo spazio attraverso le attività che

\footnotetext{
${ }_{10}$ SHELLER, Mimi, URRY, John. The new mobilities paradigm, p. 208.

11 VECCHIO, op. cit.

${ }^{12}$ FINK, Camille. More Than Just the "loser Cruiser"? An Ethnographic Study of the Social Life on Buses.

13 SHELLER, URRY, op. cit.

${ }^{14} \mathrm{NASH}$, Jeff. Bus ridings: community on wheels.

${ }^{15}$ ZURCHER, Louis A. The airplane passenger: protection of self in an encapsulated group.

16 VANNINI, Phillip. Mobile cultures: from the sociology of transportation to the study of mobilities.
} 
svolgono durante gli spostamenti, ancora di più da quando le tecnologie permettono di essere al tempo stesso sul mezzo di trasporto e "altrove".

Gli studenti sono stati stimolati a rivolgere l'attenzione al filobus come a uno spazio dove avviene la coesistenza quotidiana di gruppi molto diversi dal punto di vista sociale, culturale ed economico, cercando di comprendere forme e modalità di negoziazione quotidiana della differenza in uno spazio compresso. In questo senso, lo studio guarda anche ai temi sviluppati dal "multiculturalismo quotidiano", proponendo uno sguardo capace di osservare l'esperienza della diversità in alcuni luoghi specifici ${ }^{17}$. Senza negare la rilevanza delle norme più generali - incluse le politiche nazionali di inclusione - l'attenzione al multiculturalismo quotidiano esplora come la diversità è vissuta nelle situazioni quotidiane, focalizzandosi su come le relazioni sociali e l'identità delle persone possono essere costantemente riformulate producendo tensioni e conflitti, ma anche trasformazione e ibridazione culturale.

In linea con questa prospettiva, Amin ${ }^{18}$ sostiene che la convivenza urbana non è fatta solo di tensioni e conflitti e che gran parte della negoziazione della differenza avviene su un livello "molto locale", attraverso le esperienze quotidiane di riconoscimento e incontro. Il suo lavoro enfatizza anche la rilevanza della vivibilità degli spazi nella dimensione locale, così come delle "micro politiche dell'esistenza sociale quotidiana". I luoghi ideali per comprendere la differenza sono quelli dove le "negoziazioni prosaiche" sono obbligatorie in "micro-pubblici" come gli spazi di lavoro, le scuole, i giardini pubblici e altri spazi associativi. Amin sottolinea come le persone possano entrare in contatto e collaborare su un obiettivo comune anche in assenza di una totale accettazione dell'estraneo o un'elevata intensità di legami relazionali, dando vita a una "cultura materiale dello stare insieme" che è particolarmente evidente nei micro spazi pubblici urbani.

Le sollecitazioni derivate dalla letteratura sono state un materiale utile ad alimentare via via la discussione e il confronto tra i partecipanti, al contempo impegnati nelle prime esplorazioni sul campo e confrontati essi stessi con quella sorta di "allenamento corporeo" che è implicata nella frequentazione di un mezzo di trasporto spesso affollato oppure nel suo utilizzo in fasce orarie non usuali.

\section{La 90/91, bus degli stranieri? Migrazioni, discorso pubblico e politiche: Milano, Italia}

L'esplorazione sulla 90/91 è stata una straordinaria occasione per mettere sotto osservazione le condizioni di coesistenza in una città caratterizzata da una

\footnotetext{
17 COLOMBO, Enzo, SEMI, Giovanni (eds.). Multiculturalismo quotidiano. Le pratiche della differenza; WISE, VELAYUTHAM, op. cit.

${ }^{18}$ AMIN, Ash. Ethnicity and the Multicultural City: Living with Diversity.
} 
forte crescita della popolazione straniera e segnata da discorsi e retoriche che accompagnano i processi migratori quando diventano visibili nella scena urbana.

L'immigrazione straniera in Italia è un fenomeno recente al confronto con altri Paesi Europei e ancora oggi è spesso trattata come un fenomeno di emergenza temporanea. Gli stranieri residenti sono quasi sei milioni e costituiscono circa l'8,3\% della popolazione del paese ${ }^{19}$. I dati disponibili rivelano una presenza consistente, che per alcuni anni è stata in continuo aumento, anche se dal 2014 tale incremento è andato attenuandosi. Si tratta di un fenomeno strutturale che pone problemi di governo, in un contesto nazionale che ha sviluppato un discorso pubblico molto aggressivo su questo tema ${ }^{20}$. Negli ultimi vent'anni, a livello nazionale sono state varate ben cinque leggi sull'immigrazione, una serie di sanatorie per regolarizzare gli immigrati irregolari e diverse misure messe in atto a partire dal 2011 per far fronte a quella che viene descritta come "I'emergenza profughi": persone che fuggono da paesi politicamente instabili o in guerra. Un tema, quello del governo dell'arrivo dei rifugiati e dei profughi, che non è al centro dell'attenzione di questo saggio, ma che sicuramente contribuisce ad alimentare sullo sfondo un discorso pubblico allarmistico sull'immigrazione.

Il caso di Milano e della sua regione urbana - uno dei centri economici più importanti d'Italia - deve dunque essere inquadrato in questo contesto. Milano è una delle destinazioni primarie dei flussi migratori in Italia con una popolazione straniera che, negli anni Duemila, si è attestata attorno al 19\%. I residenti stranieri regolari sono circa 260.000, su una popolazione complessiva di un milione e trecentomila abitanti. Si tratta di una città in cui i fenomeni di polarizzazione sociale sono particolarmente intensi ${ }^{21}$ anche a fronte delle molte iniziative e politiche volte a garantire la tenuta economica della città ${ }^{22}$.

Dal 1993 al 2011, le Giunte di centro destra che hanno guidato la città hanno teso ad appiattire il tema dell'immigrazione su agende di natura securitaria. Tuttavia, anche a Milano la delega di ruoli e funzioni in materia di accoglienza e supporto ai processi di "integrazione", al volontariato, ai sindacati, alla chiesa e al terzo settore ha svolto un ruolo cruciale e ha potuto contare su una società civile particolarmente ricca che ha tradizionalmente contribuito a delineare il modello di welfare della città. L'ultima giunta di centro destra guidata da Letizia Moratti fino al 2011 si è contraddistinta per un discorso pubblico particolarmente aggressivo nei confronti dei nuovi arrivati ${ }^{23}$.

${ }^{19}$ AMBROSINI, Maurizio. Migrazioni.

${ }^{20}$ RIVERA, Annamaria. Regole e roghi. Metamorfosi del razzismo.

${ }^{21}$ CUCCA, Roberta, RANCI, Costanzo. Unequal Cities: The Challenge of Post-Industrial Transition in Times of Austerity.

22 BARBERIS, Eduardo et alii. Urban Policy on Diversity in Milan; PASQUI, Gabriele. Milano.

${ }^{23}$ ARRIGONI, Paola. Terre di nessuno. Come nasce la paura metropolitana; BRIATA, Paola. Spazio urbano e immigrazione in Italia. Esperienze di pianificazione in una prospettiva europea. 
A partire dal 2011, con una giunta guidata da un sindaco di centrosinistra, si registra un cambiamento del discorso pubblico sull'immigrazione. In termini teorici, emerge un'idea di sicurezza ad ampio spettro, attenta alla riqualificazione e alla vitalità dei territori. Ma, da un lato, manca la capacità delle politiche di convergere verso un frame condiviso e integrato, dall'altro al cambiamento di discorso pubblico non sembrano comunque corrispondere azioni di policy capaci di segnare una reale discontinuità con il passato ${ }^{24}$ con l'eccezione della politica disegnata per fare fronte all'arrivo dei profughi alla stazione centrale di Milano a partire dal $2014^{25}$.

\section{La 90/91: fa paura?}

Le linee 90 e 91 sono due filobus che, compiendo in sensi opposti lo stesso percorso, segnano come un compasso l'intera città lungo la circonvallazione esterna delineando una sorta di confine simbolico e materiale fra centro e periferia.

La circolare rappresenta una linea strategica nella città di Milano, perché collega fra loro alcune aree periferiche della città e linee di trasporto radiale, toccando zone molto diverse e alcune delle più importanti stazioni della metropolitana; svolge un servizio continuo 24 ore su 24 e I'ampiezza del servizio offerto risponde alle necessità di una grande varietà di persone: chi fa le ore piccole, chi si alza presto per andare a lavoro, venditori di rose, turisti diretti alla stazione, studenti e chi non ha dove dormire e vi passa la notte per scaldarsi.

II numero elevato di passeggeri fortemente connotato per una presenza di immigrati non passa inosservato e anzi è spesso al centro del dibattito pubblico. Inserendo "viaggio sulla 90/91" su un qualsiasi motore di ricerca nella rete, ci si accorge che la narrazione legata a questa linea richiama i termini della "paura", di una realtà "malfamata", di "inferno" e "violenza", paragonata a scenari di "guerra" con personaggi "mostruosi"26. La circolare è infatti posta all'attenzione pubblica quasi esclusivamente come oggetto di denuncia e di richiamo all'immigrazione straniera come motivo di allarme sociale. Ciononostante sono pure numerose le rappresentazioni ironiche, i progetti fotografici e artistici, i blog e le raccolte di esperienze ${ }^{27}$ volte a sfatare l'immagine prevalente del filobus.

\footnotetext{
${ }^{24}$ MARZORATI, Roberta, QUASSOLI, Fabio. Governing diversity in Milan 'città mondo': political discourse and policies towards 2015 Expo; BARBERIS, Eduardo et alii. Dealing with urban diversity.

${ }^{25}$ BOVO, LIPPI, op. cit.; BRIATA, Paola, CASTELNUOVO, Ida, COSTA, Giuliana. Teaching-in-the field in a hub accommodating migrants in transit in Milan.

${ }^{26}$ Striscia la notizia, trasmissione televisiva, Ottobre 2017; Affari italiani, quotidiano online, Ottobre 2017; Corriere della Sera, Dicembre 2009; La Repubblica, Ottobre 2017; il Giornale, Ottobre 2017; "Nell'inferno notturno dei bus 90-91", il Giornale, Ottobre 2017; il Giorno, Ottobre 2017.

27 Il milanese imbruttito, Ottobre 2017; Vice, Marzo 2015; Mostra Fotografica Ritratti, situazioni, dettagli sulla linea 90-91 lungo la circonvallazione milanese (Spazio Raw Milano, Novembre 2015); Progetto Super 9091 (all'interno di Super il festival delle periferie, Milano, 2014-2018).
} 
A fronte di un discorso pubblico che riafferma continuamente un processo di stigmatizzazione della linea, la risposta delle amministrazioni succedutesi al governo della città si è realizzata essenzialmente attorno al tema della sicurezza, con tratti più o meno evidenti fra amministrazioni di destra e di sinistra. Se la Giunta Moratti aveva esplicitamente adottato misure repressive, le cosiddette "ronde" sulla linea, la successiva Giunta Pisapia proponeva, con I'assessore al Welfare Majorino, "unità mobili sociali" con l'intento di mitigare i provvedimenti in materia di sicurezza con azioni sociali; anche l'attuale Giunta non ha mancato di affrontare il tema della 90/91 in termini di sicurezza e a Novembre 2017 proponeva di istituire un presidio permanente di security sulle linee notturne.

Una rassegna più accurata fa emergere una realtà più complessa e diversificata. Di giorno la linea viene raccontata con immagini diverse, chi denuncia la presenza di stranieri, chi riporta eventi di furti, non meno frequenti che in altri mezzi, chi invece valorizza la diversità che si concentra sul filobus. Le narrazioni sembrano convergere, con tratti più o meno ironici, nelle visioni notturne della linea, dove la diversità si disperde e la disperazione si incrocia con l'insicurezza. Viene da chiedersi quindi se il dibattito pubblico non sia esito di una sorta di sineddoche, dove una parte del racconto, quella della realtà notturna del filobus, diventa totalità e dove la 90/91 diviene integralmente oggetto di una forte stigmatizzazione. A fronte di queste osservazioni e con la volontà di investigare le peculiari condizioni di coesistenza e negoziazione della diversità, un approccio etnografico e una ricerca estensiva condotta dal gruppo di lavoro hanno contribuito ad articolare e superare l'univocità del racconto. L'esplorazione diretta e prolungata restituisce un'immagine estremamente diversificata, non solo a seconda delle ore del giorno, ma anche degli utilizzatori e dei loro comportamenti. Sguardi capaci di rivelare le potenzialità e i limiti di alcune situazioni e di arricchire la narrazione stessa, proprio perché realizzata a partire da punti di vista che sono molto diversi anche solo per i diversi background nazionali degli studenti che hanno partecipato alla ricerca.

\section{Milano vista dalla 90/91: pratiche sociali e mappe impreviste}

Alcune esplorazioni degli studenti hanno messo a tema in modo esplicito il percorso, la traiettoria, gli orari del filobus assumendolo come un punto di osservazione mobile sul contesto cittadino. Certamente questo è il caso di chi ha insistito nella sua esplorazione su alcune specifiche tratte della linea o ancora sui luoghi in cui la 90/91 fa tappa, ovvero sugli spazi delle fermate. Mesfin Alemu² - studente etiope - ha incontrato e dialogato sul bus con i suoi connazionali e con persone di origine eritrea che usano abitualmente la linea. Insieme alle

\footnotetext{
${ }^{28}$ ALEMU, Mesfin. An Ethiopian and Eritrean perspective on the 90/91 bus.
} 
testimonianze di chi racconta di aver fatto l'abitudine al fatto che i passeggeri italiani cambiano spesso di posto quando una persona di colore si siede accanto a loro, Alemu si è concentrato su un particolare tratto della linea che corre tra la fermata in prossimità della Stazione centrale e la fermata di Piazzale Susa. Sono questi i due estremi tra i quali si muovono i migranti provenienti dal corno d'Africa. Stazione Centrale è punto di approdo e di raccolta, Piazzale Susa è invece il luogo d'interscambio dove i passeggeri trasbordano sul bus numero 38 che conduce al centro di prima accoglienza di Via Corelli. E i grandi spazi aperti di Piazzale Susa diventano luogo di incontro, di scambio d'informazioni e di servizi informali per uno specifico gruppo di immigrati che cerca orientamento nei meandri delle procedure burocratiche per gli immigrati.

Un'intensa osservazione dei frequentatori della linea 90/91 e un piccolo incidente occorso durante la notte ha portato Szymon Michalski ${ }^{29}$ - studente polacco - a profilare una specifica utenza: quella dei giovani che si muovono a tarda notte tra i locali e i club della città. Durante un primo viaggio Michalski e un gruppo di amici salgono sul mezzo con gli avanzi di una festa diretti a un locale. I bicchieri di cocktails destano l'attenzione di un uomo musulmano che rimprovera il mancato rispetto delle regole di Ramadan sotto gli occhi dei suoi figli piccoli, aprendo a uno scambio serrato e acceso con i ragazzi già un poco alticci. Questa situazione, un incidente, porta Michalski a soffermarsi sulla frequenza con cui la notte si consumano cibi e bevande sulla 90/91. Insieme a una rassegna fotografica che documenta ricorrenza e varietà di queste situazioni, con il supporto di Tripadvisor, l'esito del lavoro di ricerca è una mappa che mette in evidenza come molti dei luoghi di aggregazione e intrattenimento più popolari tra i giovani a Milano siano di fatto attestati in prossimità della circonvallazione esterna. Ci si sposta spesso da un locale ad un altro, si usa la 90/91 quale risorsa disponibile anche nel pieno della notte per rientrare verso casa. Atmosfere, pratiche, gruppi di amici trasbordano letteralmente dallo spazio dei locali allo spazio del bus che si configura come una sorta di "prolungamento" o di ponte tra i locali stessi.

Chi ha concentrato la propria indagine sugli spazi delle fermate ha spesso individuato piste di grande interesse. Le fermate sono spazi di sosta, di attesa, sospensioni temporali lungo un percorso. In modo quasi paradossale, "per muoversi è necessario fermarsi e sostare" scrive lo studente francese Victor $\operatorname{Gardin}^{30}$. Alle fermate si gioca una relazione stretta, ma assai articolata, tra lo spazio pubblico della fermata e lo spazio pubblico del bus. Alle fermate l'immobilità, il sostare inoperoso nell'attesa e la promiscuità producono quale effetto un potenziamento del controllo sociale e dell'attenzione al contesto. La

\footnotetext{
${ }^{29}$ MICHALSKI, Szymon. The taste of 90/91.

${ }^{30}$ GARDIN, Victor. Waiting for the 90/91.
} 
prossemica fa il suo gioco e le distanze personali iniziano a entrare in tensione prima ancora della "compressione" che si produrrà sul bus.

I percorsi di osservazione che Gieles Kinget - studente belga di cui riportiamo in queste pagine le fotografie (fig. 2-5) - ha tracciato a partire dalla sua osservazione di alcune fermate, sembrano confermare quanto la 90/91 sia un confine simbolico e materiale tra le parti più centrali e quelle più periferiche della città. Partendo da un'osservazione di dettaglio, Kinget ${ }^{31}$ ha rilevato e documentato volantini, minuscoli annunci e post-it di carta che vengono affissi a pali, pensiline e strutture verticali in prossimità delle fermate. Un uso estensivo della comunicazione tradizionale che pare una sorta di sovversione rispetto all'intensità del flusso di informazioni che circola in città per via digitale e che dissemina, mette in scena e rende visibili pratiche che affollano un mercato informale. Una lettura e un'analisi più attenta ha selezionato due offerte specifiche, componendo una panoramica sulla domanda e offerta di posti di lavoro a bassa qualificazione (essenzialmente nel campo dei servizi) e di alloggi o posti letto in locazione a canoni accessibili. La trasposizione delle informazioni raccolte sulla planimetria della città fa emergere una mappa dei luoghi del mercato informale di case e posti di lavoro sistematicamente esterni all'anello della 90/91. Una conferma dell'assetto fortemente monocentrico di Milano, in cui i valori sono tendenzialmente distribuiti secondo una scala decrescente dal centro alla periferia.

\section{Milano sulla 90/91: la compressione spaziale come lente sulla Cosmopolis}

Andreas De Mesmaeker ${ }^{32}$, studente belga fa della sua scarsa conoscenza dell'italiano un punto di forza per la sua osservazione partecipante, concentrando l'attenzione sul paesaggio sonoro del bus. Il rumore del filobus è per molti versi inconfondibile e tipico rispetto agli altri bus della città per via della trasmissione elettrica, ma sono i suoni, le voci e i rumori a bordo a costituire un materiale straordinario. "Ragazza... tiiiiing; Ragazza ... Tiiing" è una sequenza di suoni metallici e di parole ripetute da un passeggero che sta seguendo un corso di italiano online utilizzando uno smartphone: ogni volta che la risposta è esatta, lo smartphone vibra, suona e permette di passare a un livello di apprendimento più elevato. II lavoro di ricerca descrive con immagini che richiamano le forme di rappresentazione dei futuristi uno spazio pubblico quanto mai in movimento, in cui la frammistione di suoni e rumori rasenta la cacofonia in un spazio che è spesso compresso e sovraffollato. Eppure, De Mesmaeker ci restituisce l'immagine di uno spazio in cui il tempo non è mai

\footnotetext{
31 KINGET, Gieles. Paperwork(s).

${ }^{32}$ DE MESMAEKER, Andreas. A Waste of Time? A Sound-Reconstruction of How Bus Users in Milan Spend Their Time While Travelling.
} 
sprecato, in cui i singoli trovano spesso modo di impegnare lo spazio/tempo durante il proprio tragitto per pratiche minori.

Comportamenti, attitudini e profili dei passeggeri che affollano lo spazio della 90/91 sono stati oggetto dell'osservazione di Irem Okandam e Eugenia larlori, rispettivamente studentessa turca e italiana. Riprendendo la definizione di $\mathrm{Amin}^{33}$ di "animali urbani", la loro ricerca ha individuato e tratteggiato alcuni profili tipici. Le categorie di riferimento non sono quelle consuete - etnia, età, sesso. Riprendendo la metafora della catena alimentare entro la quale si individuano gerarchie di prede e predatori, la mappatura dei passeggeri evidenzia le più comuni espressioni di territorialità. $\mathrm{Ci}$ sono passeggeri che tendono esplicitamente ad isolarsi, ad abbassare lo sguardo e a circoscrivere il proprio spazio individuale (defenders e isolators), altri che sono careless e indifferenti, altri che risultano più invasivi anche solo perché si muovono in gruppo (with company) e altri invece che hanno più evidenti comportamenti dominanti che li connotano quali invaders e predators ${ }^{34}$. La ricerca mette in evidenza il modo in cui una varietà di comportamenti individuali contribuisce a fare della 90/91 uno spazio più o meno pubblico, in cui percezioni, posizionamenti, contese e negoziazioni vedono in scena attori che sono riconducibili ad alcuni ruoli che possono essere classificati in quanto ricorrenti. Dove inizia e finisce la mia libertà (d'uso) rispetto al modo in cui io stesso/a limito la libertà d'uso degli altri? Questa è una domanda fondamentale nella convivenza tra strangers.

A partire dall'ironica differenza fra la realtà dei bus italiani e quelli tedeschi, Nina Pfeil, studentessa di Amburgo, pone la propria attenzione su uno spazio particolare del bus, l'area di ingresso/uscita, facendone una lente per un'osservazione più ampia. Nell'area in prossimità delle porte i passeggeri dei bus italiani sembrano essere soggetti ad una sorta di irresistibile magnetismo, the door appeal. Maggiore il numero di persone, più forte sembra essere la necessità di assicurarsi un proprio spazio, a tutti i costi. Entro questa norma, uno sguardo attento evidenzia alcune peculiarità che contribuiscono a caratterizzare questo spazio rispetto al resto del bus: in primo luogo, la presenza di una linea sottile fra un generico atteggiamento di cortesia e l'insorgere di un istinto di sopravvivenza, una lotta per il proprio spazio, in prossimità delle porte. Nelle conclusioni, con sua stessa sorpresa, Pfeil $^{35}$ segnala: nonostante le differenze fra i passeggeri siano molte, nella 90/91 la forza del door-appeal è tale da far prevalere un'unica "cultura del bus" che accomuna tutti, a prescindere dalle condizioni culturali e sociali. Entro lo spazio pubblico compresso del bus, il lavoro evidenzia quindi uno spazio ulteriormente compresso, dove i passeggeri

\footnotetext{
33 AMIN, Land of Strangers, op. cit.

34 IARLORI, Eugenia, OKANDAM, Irem. Personal I Public Space.

35 PFEIL, Nina. The Door-Appeal.
} 
non possono che esercitarsi in micro-interazioni e nel farlo dimostrano di condividere un unico comportamento culturale.

Come abbiamo già anticipato, il "lasco", ovvero la possibilità che si diano movimento, pratiche d'uso, e lo stesso accesso a un mezzo pubblico sono fortemente condizionati da una pluralità di fattori che possono produrre vera e propria esclusione. Vinita Wagh, indiana, mai ha anche solo immaginato di salire su un autobus pubblico a Pune: "non sarei durata neppure un giorno". A Milano, scopre la 90/91 a due passi da casa. Le appare quasi come un miraggio: le fermate tutte uguali, standardizzate e confortevoli, il mezzo capiente e scarsamente frequentato. Lo spazio delle fermate, in India descritto come un corpo unico di una massa compatta di persone in attesa, a Milano è uno spazio in cui si ha tutto il tempo e lo spazio per osservarsi vicendevolmente, per soffermarsi sui dettagli, le tracce sul terreno, l'abbigliamento e gli sguardi dei futuri compagni di viaggio. II suo è un racconto profondamente ironico che relativizza il discorso stigmatizzante sviluppato dai media. Con grande umorismo, ne descrive i tratti di ordinarietà per poi evidenziare le increspature sulla superficie piatta. "La vita nella sfera pubblica è fortemente anonima, ma evitare le altre persone richiede molti sforzi" ${ }^{\prime \prime 6}$. Il lasco, la relativa rarefazione dello spazio apre infatti a infrazioni della sfera personale che possono risultare intrusivi. Wagh descrive la sua progressiva esposizione a situazioni che si sono rivelate imbarazzanti per le domande dirette che le sono state rivolte (cosa che nel suo paese mai difficilmente avrebbe luogo). Spesso è stata oggetto di interesse e curiosità: "da quale paese vieni?", "da quanto sei a Milano?", "dove abiti?". "Mai i locali e mai giovani studenti. I più interessati a parlare con la sottoscritta paiono i non-nativi di mezza età" ${ }^{\prime \prime 7}$. L'irruenza delle domande arriva ad essere quanto mai intrusiva e sconvolgente il giorno in cui, quale prima domanda, le viene chiesto: "a quale religione appartieni?". Se a Milano si tratta di una questione tenue, in India questa è materia di violento conflitto aperto.

Il paragone con un diverso paese d'origine, ancora una volta, riesce a restituire una lettura alternativa della circolare 90/91. Bilal El Ghoul, non può prescindere dallo sviluppare la propria ricerca a partire da una riflessione sul contesto libanese e sull'accezione che il bus e il trasporto pubblico hanno a Beirut, dove con l'attacco a un bus ebbe inizio nel 1976 la guerra civile e dove oggi questo mezzo continua ad esserne il simbolo. Se in qualche modo i conducenti libanesi seguono delle linee, ogni corsa è continuamente soggetta a variazioni, che l'autista compie per accorciare la strada, fare una sosta o intercettare più passeggeri. Non esistono punti definiti per le fermate che cambiano continuamente nel tempo e nello spazio, a discrezione dei passeggeri.

\footnotetext{
${ }^{36}$ WAGH, Vinita. Not a bus story (I am kidding, it is).

${ }^{37}$ Ibidem.
} 
A fronte della "visibile irregolarità" del sistema, in cui orari e percorsi sono quasi esclusivamente determinati dalla componente umana, El Ghoul ${ }^{38}$ concentra la sua osservazione sulle "invisibili irregolarità" del più strutturato trasporto milanese: I'apertura delle porte per passeggeri ritardatari, contrattempi dovuti a richieste d'informazioni all'autista o a repentini attraversamenti della strada. La 90/91 viene narrata come essenzialmente ordinaria, a tal punto da voler valorizzare quelle micro variazioni che arricchiscono l'esperienza di trasporto e diventano spazio prezioso di espressione personale.

Sara Eltokhy, studentessa egiziana, conduce una ricerca dove la propria esperienza personale gioca un ruolo fondamentale e in cui fin da subito mette in discussione la nozione di cosmopolitan canopy applicata al bus. "Quando si prende la 90/91 per la prima volta, ci si sente come se non si fosse più in Italia; persone di etnie, razze e classi sociali diverse, tutti nello stesso ambiente pubblico formano una notevole mescolanza" ${ }^{\prime 39}$, a prima vista la circolare viene percepita come un ricco spazio di coesistenza e interazione leggera sulla base di regole civili. La ricerca però approfondisce l'osservazione ed evidenzia un secondo livello di maggiore complessità, in cui reti sovrapposte di individui si intrecciano. Uomini egiziani le offrono ripetutamente il proprio posto, secondo l'abitudine in Egitto, ma non lo fanno con altre donne nel bus; una signora la spinge aggressivamente per aver invaso il proprio spazio personale, "lo avrebbe fatto se non avessi avuto il velo?". Emerge così un mosaico di "micro-comunità" che velocemente si formano e si dissolvono nello spazio del bus, non su una base esclusivamente etnica, ma anche culturale, linguistica, di nazionalità e sociale. Il lavoro si conclude con l'osservazione dei passeggeri di colore, dei loro comportamenti e spostamenti nel bus. Eltokhy sostiene che nella gran parte dei casi questi passeggeri tendano a raggrupparsi nel retro del bus e lascia aperta una questione di notevole significato, inevitabilmente rimandando ad un'immagine di segregazione. Proprio per questa volontà di toccare temi tanto importanti il lavoro assume un importante significato, esprimendo la necessità di tornare a trattare certe questioni e metterle in discussione.

\section{Conclusioni}

Torniamo alle domande di ricerca proposte in apertura. Innanzi tutto, è possibile considerare la 90-91 come un cosmopolitan canopy, un luogo dove "la manifestazione dell'accettazione pubblica di tutti da parte di tutti è particolarmente intensa, diventando una delle caratteristiche che definiscono un luogo" $" 40$ ?

\footnotetext{
${ }^{38}$ EL GHOUL, Bilal. Invisible lines.

${ }^{39}$ ELTOKHY, Sara. Ghettos on the go.

40 ANDERSON, op. cit., p. 3.
} 
$E^{\prime}$ utile soffermarsi sui lavori che hanno mirato a comprendere come avviene la negoziazione della differenza e come si riflette nell'uso dello spazio del filobus. Le risposte emerse sono molto articolate: non sono né univoche, né scontate, anche all'interno di ogni singolo percorso di osservazione. I risultati di ricerca ci paiono confermare la fertilità del posizionamento "sul campo" - osservare la convivenza tra estranei nell'ambito di uno spazio pubblico compresso - così come della scelta metodologica di moltiplicare gli sguardi, affidando una parte significativa di osservazione a studenti a uno stadio ormai maturo del loro percorso di formazione e provenienti da paesi diversi.

La compressione si rivela un amplificatore per molti versi straordinario per osservare le relazioni nello spazio, cosa fa eccezione e cosa costituisce la norma. La 90/91 è una "conduttura di persone" che costringe allo stare assieme anche per brevi tratti, ma dove, contrariamente agli aerei per i quali era stata mobilitata questa immagine, la compresenza tra individui sconosciuti e differenti è poco mediata dalle regole formali - che pure esistono, sono molto dettagliate ed esposte pubblicamente nel filobus.

Un viaggio sulla 90/91 non configura un'esperienza culturale neutra, ma si tratta di un luogo di accettazione pubblica dove tutti accettano tutti? A prima vista l'uso intenso di questo mezzo da parte di popolazioni così diversificate (inclusi i gruppi privilegiati della città) porta a rispondere in modo affermativo, anche se il lavoro attento degli studenti e il loro sguardo, a volte "straniero", talvolta anche spiazzato se non inizialmente smarrito, racconta di un luogo di tolleranze e intolleranze, curiosità anche invadenti verso chi è diverso, solidarietà etnica e non solo. Un mondo nel quale entrano altri mondi - si pensi solo a quanto gli smartphone a volte amplifichino la compresenza di lingue diverse: sulla 90/91 non solo si parlano molte lingue, ma è anche un luogo da dove si parla con tutto il mondo e, al tempo stesso, s'impara l'italiano. Ma è importante anche non negare il lato più oscuro dell'incontro quotidiano con la differenza: soprattutto chi non ha la pelle bianca o porta un velo islamico, ha registrato con grande evidenza come il filobus sia anche un luogo dove si manifestano, in termini fisici, spaziali e sociali, forme di razzismo.

Forse la 90/91 non è ancora una cosmopolitan canopy come quelle descritte da Anderson, ma è certamente una "palestra" per allenarsi alla convivenza in una città oggi cosmopolita come Milano. Molte osservazioni restituiscono l'immagine di un esercizio difficile, non scontato: la convivenza si conferma anche come un esercizio del corpo. Se "evitare le persone richiede molti sforzi", stare assieme è anche una questione di allenamento corporeo, un'abitudine a suoni, odori, abitudine (e indifferenza) a colori della pelle diversi, modalità eterogenee di intendere cos'è la prossimità o la distanza, la buona e la "mala-educazione". Una molteplicità di "animali urbani" si appropria dello spazio in modo più o meno invasivo fino a diventare 
predatore, ma delineando usi che sovvertono le più tradizionali categorie di osservazione della città. Su questo fronte si delinea un terreno di ricerca aperto che va oltre la razza, la religione e l'etnia e ci porta a interrogarci su dove inizi e finisca la libertà (d'uso) di ciascuno nel momento in cui un comportamento limita la libertà d'uso degli altri. Questa è una domanda fondamentale nella convivenza tra strangers.

In che modo, le esplorazioni realizzate possono essere utili a Milano? La risposta non è scontata, ma pensiamo che la repressione - le ronde notturne, i servizi di security - concentrino l'attenzione su pratiche e sovversioni che avvengono in tempi e orari davvero molto limitati. La 90/91 si presenta come una lente formidabile per guardare alla cultura materiale dello stare insieme, così come per comprendere quali possono essere le condizioni, le sfide e i limiti per costruire quella "politica dello stare insieme" evocata da Amin ${ }^{41}$ per contrastare la diffidenza verso l'estraneo.

Le ronde ci conducono a un altro tema cruciale: quello dello stigma che grava sulla 90/91 e che spesso di traduce in azioni pubbliche di natura securitaria ed emergenziale. Il lavoro sul campo invita a riflettere su quanto un'immagine stigmatizzata sia il prodotto di un'idea che sia possibile definire una "normalità assoluta" e che esistano luoghi come la 90/91 dove tale normalità viene abitualmente sovvertita - e lo stigma si basa su un'amplificazione delle sovversioni. Una sineddoche, abbiamo detto: le popolazioni marginali che $d^{\prime}$ inverno trovano riparo sul filobus definiscono un'immagine che rimbalza nei media e vale per tutte le stagioni: d'estate come d'inverno, di giorno come di notte. La normalità è fatta anche di molte eccezioni, compresa la tolleranza di chi produce un'azione pubblica che al momento non ha la forza materiale e politica di dare un tetto dignitoso a tutti, salvo poi reagire con repentine e discontinue azioni di natura securitaria nei momenti in cui il dibattito si fa più acceso e le luci della città sono puntate sulla 90/91.

Il confronto con una classe internazionale di studenti è stato anche una grande occasione di apprendimento per comprendere come la normalità non possa che essere definita in base al contesto: se gli articoli di giornale descrivono un viaggio sulla 90/91 come un "andare in guerra", c'è chi ci ricorda che i bus per le strade di Beirut sono il simbolo di una guerra lunga e vera, così come chi racconta con ironia la quotidiana conquista di spazio minimo vitale alle fermate e sui bus che trasportano la massa umana di Pune. Un invito quindi anche a sdrammatizzare e, al tempo stesso, a non pensare solo al dentro e al fuori del "nostro" filobus. Se lo studio sui bus di Los Angeles si chiedeva quanto lo stigma fosse generato da un confronto tra la visione che chi usa l'autobus normalmente e chi no, questo lavoro ha permesso di ampliare la nozione del

${ }^{41}$ AMIN, Land of Strangers, op. cit. 
fuori, smontando una visione stigmatizzante attraverso un allargamento dei confini e dei punti di osservazione. Allargare sempre lo sguardo. Forse anche questa è una responsabilità politica e intellettuale di chi si occupa di formazione, ma anche di chi promuove politiche pubbliche in contesti di diversità.

Infine, siamo partiti da un filobus, ma ci sembra che questa osservazione di dettaglio ci permetta di dire anche qualcosa su che tipo di città sia oggi Milano. L'apparato iconografico fornito da tutti gli studenti ha arricchito con immagini suggestive e per molti versi straordinarie i testi scritti di cui abbiamo raccontato. Nella ristrettezza degli spazi, abbiamo fatto la scelta di presentare le fotografie realizzate da Gieles Kinget (Fig. 2-5) perché ci sembra che, nel loro guardare a una cosa molto minuta, più di altre raccontino anche cos'è oggi Milano. I post-it e i volantini alle fermate della 90/91 rivelano le tracce della città informale al servizio di poveri e immigrati. Seguire e mappare queste tracce significa percorrere una città che si trova tutta al di fuori della circolare esterna. Milano si conferma come una città monocentrica dove i valori sono distribuiti secondo una scala decrescente dal centro alla periferia. La linea della 90/91 appare come un anello di congiunzione, che mentre "lega" e inanella tappe in successione lungo la sua linea circolare, segna e traccia legami e connessioni verso le porzioni esterne della città, marcando una distinzione territoriale che appare netta ed evidente e che, a fronte di una corrente retorica sui tratti di policentrismo guadagnati dalla regione urbana "che resiste alla crisi", sollecita una discussione rispetto al permanere della circonvallazione esterna quale linea di demarcazione tra valori, pratiche e popolazioni.

Figura 2 - Passeggeri della linea 90/91

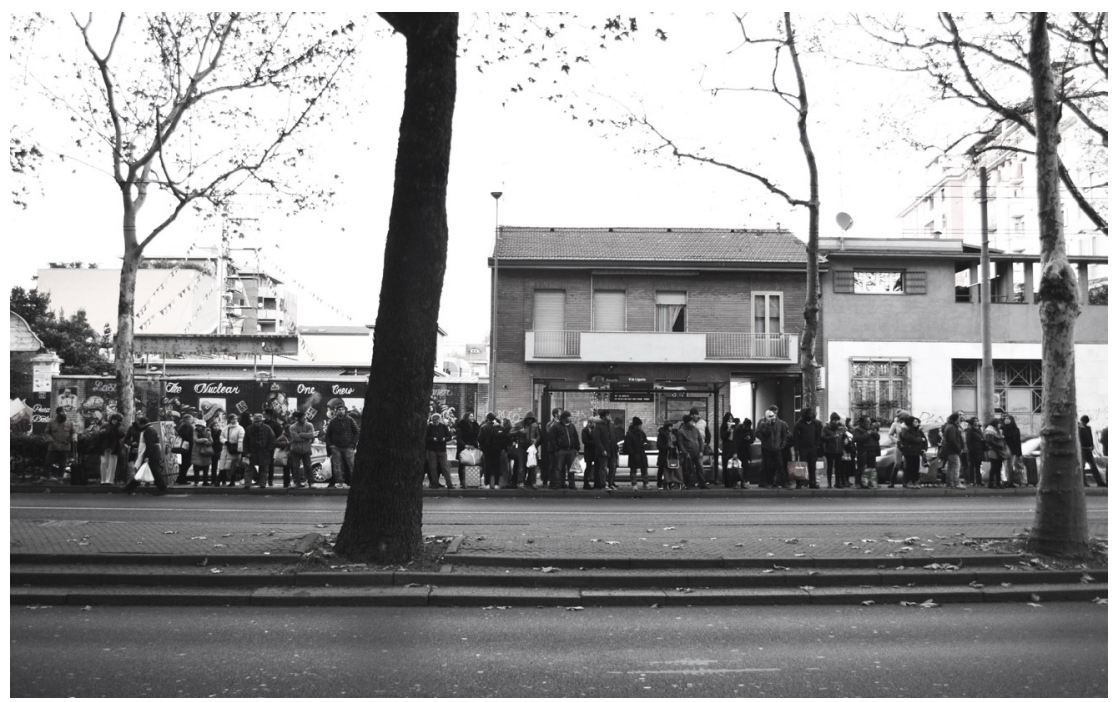

Fonte: KINGET, Gieles, 2018. 
Figura 3 - Paperwork(s) \#1

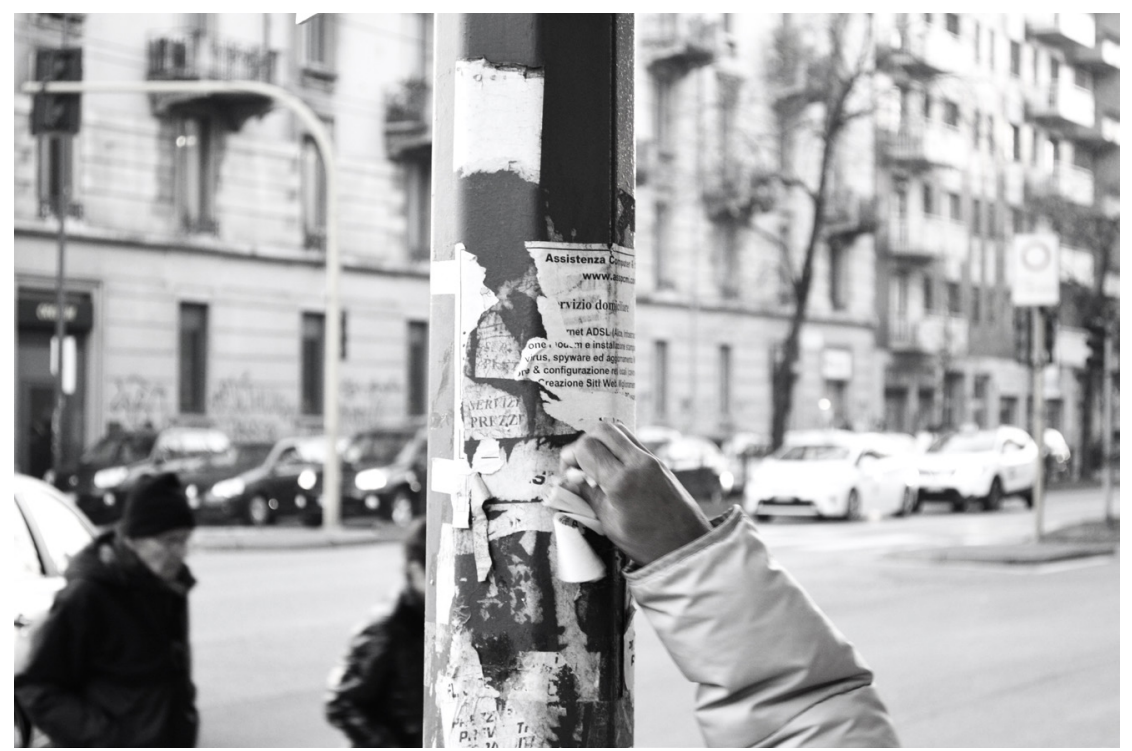

Fonte: KINGET, Gieles, 2018.

Figura 4 - Paperwork(s) \#2

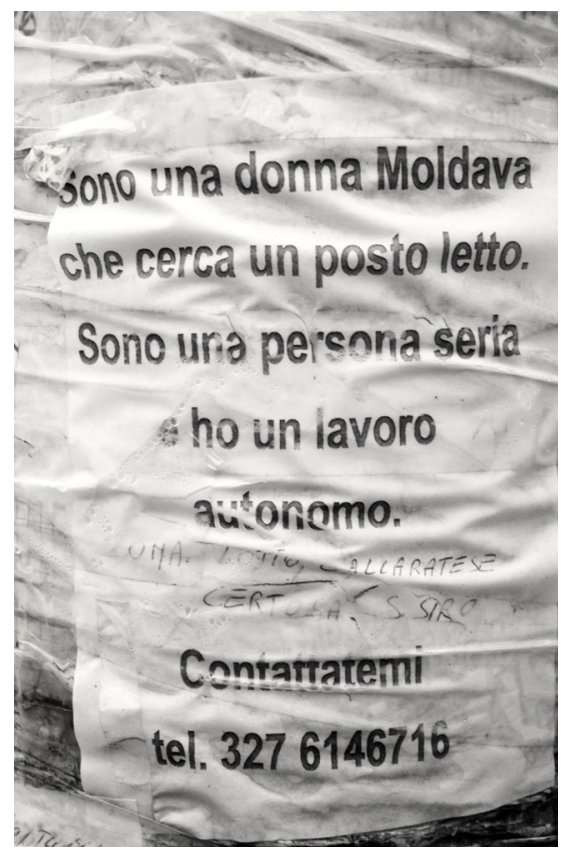

Fonte: KINGET, Gieles, 2018.
Figura 5 - Paperwork(s) \#3

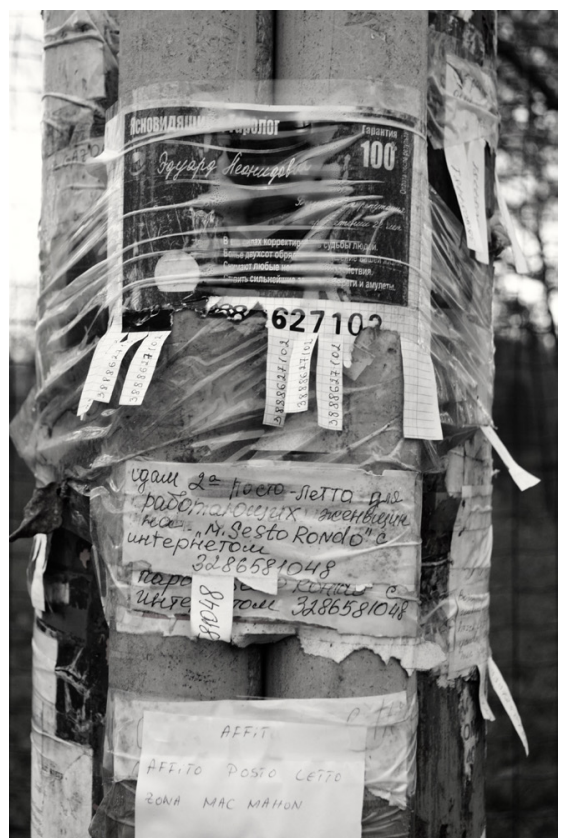

Fonte: KINGET, Gieles, 2018. 


\section{Bibliografia}

ALEMU, Mesfin. An Ethiopian and Eritrean perspective on the 90/91 bus. In BRIATA, Paola; BRICOCOLI, Massimo; BOVO, Martina (eds.). Report of the Urban Ethnography Course 2016-2018. Master of Science in urban Planning and Policy Design, Politecnico di Milano, 2018.

AMIN, Ash. Ethnicity and the Multicultural City: Living with Diversity. Environment and Planning A Economy and Space, v. 34, n. 6, 2002, p. 959-980.

AMIN, Ash. Land of Strangers. Cambridge: The Polity Press, 2012.

AMBROSINI, Maurizio. Migrazioni. Milano: Egea, 2017.

ANDERSON, Elijah. The Cosmopolitan Canopy. Race and Civility in Everyday Life. New York, London: W.W. Norton \& Company, 2011.

ARRIGONI, Paola. Terre di nessuno. Come nasce la paura metropolitana. Milano: Melampo, 2011.

BARBERIS, Eduardo; KAZEPOV, Yuri; ANGELUCCI, Alba. Urban Policy on Diversity in Milan. 2014. Disponibile su: <https://www.urbandivercities.eu/milan>.

BARBERIS, Eduardo; ANGELUCCI, Alba; JEPSON, Ryan; KAZEPOV, Yuri. Dealing with urban diversity. The case of Milan. 2017. Disponibile su: <https://www. urbandivercities.eu/milan/>.

BRIATA, Paola; BRICOCOLI, Massimo; BOVO, Martina (eds.). Report of the Urban Ethnography Course 2016-2018. Master of Science in urban Planning and Policy Design, Politecnico di Milano, 2018.

BOVO Martina; LIPPI, Chiara. The Receptive City. Tesi di laurea Magistrale in Architettura, Scuola AUIC, Politecnico di Milano, 2017.

BRIATA, Paola. Spazio urbano e immigrazione in Italia. Esperienze di pianificazione in una prospettiva europea. Milano: FrancoAngeli, 2014.

BRIATA, Paola; CASTELNUOVO, Ida; COSTA, Giuliana. Teaching-in-the field in a hub accommodating migrants in transit in Milan. Paper presentato all'AESOP annual congress Spaces of dialog for places of dignity. Lisboa, 11-14 Luglio, 2017. Disponibile su: <https://re.public.polimi.it/retrieve/ handle/11311/1036484/238020/LISBONA.pdf>.

CAPONIO, Tiziana. Città italiane e immigrazione. Discorso pubblico e politiche a Milano, Bologna e Napoli. Bologna: il Mulino, 2006.

COLOMBO, Enzo; SEMI, Giovanni (eds.). Multiculturalismo quotidiano. Le pratiche della differenza. Milano: FrancoAngeli, 2007.

CUCCA, Roberta; RANCI, Costanzo. Unequal Cities: The Challenge of Post-Industrial Transition in Times of Austerity. Routledge, 2017.

DE MESMAEKER, Andreas. A Waste of Time? A Sound-Reconstruction of How Bus Users in Milan Spend Their Time While Travelling. In BRIATA, Paola; BRICOCOLI, Massimo; BOVO, Martina (eds.). Report of the Urban Ethnography Course 20162018. Master of Science in urban Planning and Policy Design, Politecnico di Milano, 2018. 
EBERT, Clara. Bus: sharing stories. In BRIATA, Paola; BRICOCOLI, Massimo; BOVO, Martina (eds.). Report of the Urban Ethnography Course 2016-2018. Master of Science in urban Planning and Policy Design, Politecnico di Milano, 2018.

EL GHOUL, Bilal. Invisible lines. In BRIATA, Paola; BRICOCOLI, Massimo; BOVO, Martina (eds.). Report of the Urban Ethnography Course 2016-2018. Master of Science in urban Planning and Policy Design, Politecnico di Milano, 2018.

ELTOKHY, Sara. Ghettos on the go. In BRIATA, Paola; BRICOCOLI, Massimo; BOVO, Martina (eds.). Report of the Urban Ethnography Course 2016-2018. Master of Science in urban Planning and Policy Design, Politecnico di Milano, 2018.

FERRARO, Giovanni. Rieducazione alla speranza. Patrick Geddes Planner in India 1914-1924. Milano: Jaca Book, 1998.

FINK, Camille. More Than Just the "loser Cruiser"? An Ethnographic Study of the Social Life on Buses. Los Angeles: University of California, 2012.

GARDIN, Victor. Waiting for the 90/91. In BRIATA, Paola; BRICOCOLI, Massimo; BOVO, Martina (eds.). Report of the Urban Ethnography Course 2016-2018. Master of Science in urban Planning and Policy Design, Politecnico di Milano, 2018.

GEDDES, Patrick. Cities in Evolution. London: William \& Norgate Ltd, 1948.

IARLORI, Eugenia; OKANDAM, Irem. Personal I Public Space. In BRIATA, Paola; BRICOCOLI, Massimo; BOVO, Martina (eds.). Report of the Urban Ethnography Course 2016-2018. Master of Science in urban Planning and Policy Design, Politecnico di Milano, 2018.

JAIN, Juliet. The making of mundane bus journeys. In VANNINI, Phillip (ed.). The cultures of alternative mobilities: routed less travelled. Burlington: Ashgate, 2009.

KINGET, Gieles. Paperwork(s). In BRIATA, BRICOCOLI, BOVO (eds.), op. cit.

MARZORATI, Roberta; QUASSOLI, Fabio. Governing diversity in Milan 'città mondo': political discourse and policies towards 2015 Expo. Paper presentato alla Conferenza RC21 The ideal city: between myth and reality, Urbino, 27-29 Agosto 2015.

MICHALSKI, Szymon. The taste of 90/91. In BRIATA, Paola; BRICOCOLI, Massimo; BOVO, Martina (eds.). Report of the Urban Ethnography Course 2016-2018. Master of Science in urban Planning and Policy Design, Politecnico di Milano, 2018.

$\mathrm{NASH}$, Jeff. Bus ridings: community on wheels. Journal of contemporary ethnography, v. 4, n. 1, 1975, p. 99-124.

PASQUI, Gabriele. Milano. II Mulino. Viaggio in Italia, v. 6, 2017, p. 1045-1050.

PFEIL, Nina. The Door-Appeal. In BRIATA, Paola; BRICOCOLI, Massimo; BOVO, Martina (eds.). Report of the Urban Ethnography Course 2016-2018. Master of Science in urban Planning and Policy Design, Politecnico di Milano, 2018.

PISCITELLI, Paola. Mobile Urbanity in Southern Africa. In PETRILLO, Agostino; BELLAVITI, Paola (eds.). Sustainable Urban Development and Globalization. Springer, 2018, p. 33-47.

RIVERA, Annamaria. Regole e roghi. Metamorfosi del razzismo. Bari: Dedalo, 2009. 
SHELLER, Mimi; URRY, John. The new mobilities paradigm. Environment and Planning A, v. 38, n. 2, 2006, p. 207-226.

SIMONE, AbdouMaliq. People as Infrastructure: Intersecting Fragments in Johannesburg. Public Culture, v. 16, n. 3, 2004, p. 407-429.

VANNINI, Phillip. Mobile cultures: from the sociology of transportation to the study of mobilities. Sociology compass, v. 4, n. 2, 2010, p. 111-121.

VECCHIO, Giovanni. Democracy on the move? Bogotá's urban transport strategies and the access to the city. City, Territory and Architecture, v. 4, n. 15, 2017. Disponibile su: < https://link.springer.com/article/10.1186/s40410-017-0071-3>.

WAGH, Vinita. Not a bus story (I am kidding, it is). In BRIATA, Paola; BRICOCOLI, Massimo; BOVO, Martina (eds.). Report of the Urban Ethnography Course 20162018. Master of Science in urban Planning and Policy Design, Politecnico di Milano, 2018.

WISE, Amanda; VELAYUTHAM, Selvaraj (eds.). Everyday multiculturalism. Basingstoke: Palgrave Macmillan, 2009.

ZURCHER, Louis A. The airplane passenger: protection of self in an encapsulated group. Qualitative sociology, v. 1, n. 3, 1979, p. 77-99.

Articolo ricevuto il 31.01.2018 Accettato per la pubblicazione il 06.03.2018 Received for publication in January 315t, 2018 Accepted for publication in March 06 ${ }^{\text {th }}, 2018$

ISSN impresso 1980-8585

ISSN eletrônico 2237-9843

http://dx.doi.org/10.1590/1980-85852503880005210 